\title{
PODER LOCAL Y EVOLUCIÓN SOCIAL EN EL PAÍS VALENCIANO DEL SIGLO XIX
}

por

\section{MANUEL MARTí*}

Universitat de València - Estudi General

RESUMEN: En este articulo se debaten los logros e insuficiencias de los estudios sobre el poder local en el País Valenciano del siglo XIX. La radicalidad de la muptura introducida por el proceso revolucionario liberal es su punto de partida. Los mecanismos de cambio social puestos en marcha por esa ruptura no dejaron de funcionar ni siquiera tras el fracaso de la experiencia democrática iniciada en 1868. Para discutir ambas afirmaciones (no siempre compartidas por la bistoriografia española) se propone el análisis de casos locales durante periodos cronológicamente amplios y una aproximación social a la politica, con especial énfasis en las actitudes y acciones de los grupos subalternos.

\section{Palabras Clave: Siglo XIX, España, Poder local, Clases sociales.}

ABSTRACT: This paper offers the acbievements and lacks of the studies on local power in the ancient Kingdom of Valencia during the XIXth century. The breakdown of the Ancien Regime by the liberal revolution is the point of departure. The process of social change started whith the revolution continued ever after the failure of the democratic experience thet begun at 1868. To discuss these topics (not always shared by spanish bistorians), it would be proposed the analysis of local cases during long-termed periods and a social approach to politics, specially referred to attitudes and action of lower social groups.

\section{KEYWORDS: XIXth Century, Spain, Local power, Social Classes.}

* El autor participa en el proyecto DGICYT PB93-0358-C02-02. Esta colaboración se inscribe también en el proyecto DGICYT PB94-1120 dirigido por el Dr. Carasa.

Hispania, LIX/1, núm. 201 (1999) 51-58 
Los estudios sobre el poder local representan, en el País Valenciano, una contribución parcial al análisis de la peculiar evolución de su sociedad durante el siglo XIX. Este carácter limitado y fragmentado puede explicarse, en parte, por la atención preferente a otros temas y periodos, tal y como se expondrá más adelante. Pero también tiene su origen en las preferencias teóricas y metodológicas de algunos sectores de la historiografía valenciana. Así, para aquellos autores que utilizan el paradigma de la modernización como clave explicativa, las etapas anteriores a las décadas finales de la pasada centuria carecen, por lo visto, de interés, puesto que el inicio del proceso modernizador se fija, bastante arbitrariamente, en el periodo crítico de la Restauración canovista. Desde ese sesgado punto de vista, el siglo XIX aparece caracterizado por una imagen global de estancamiento y pasividad escasamente alterada por las agitaciones politicas y sociales de sus primeras décadas. En el campo de la política local, este enfoque, dado a la comprensión de la política como un proceso en buena medida marcado por los valores, actitudes e iniciativas de las élites, sólo ofrece, en el caso de la Restauración, análisis precisos de determinados aspectos de ciertas redes caciquiles '. Tampoco una cierta perspectiva que se reclama depositaria legítima de la tradición marxista arroja nueva luz sobre la evolución de las estructuras políticas locales ${ }^{2}$.

Sin embargo, el País Valenciano sí cuenta con una ya larga tradición de estudios de ámbito territorial restringido, que arrancan, en su mayor parte, del largo y fecundo debate sobre la peculiaridad nacional de los valencianos y se centran, también en gran proporción, la cuestión de la crisis del Antiguo Régimen y sus consecuencias sociales y económicas ${ }^{3}$. Este punto de arranque impone, para cualquier análisis del problema del poder local, una perspectiva de larga duración de la que se beneficia enormemente. Esa perspectiva parte de la caractetización abiertamente rupturista del proceso de revolución liberal en el

1 De ello tratan los estudios de A. YANINI, como El caciquisme (Valencia, 1984) o «Funcionamiento del sistema político y estructura de poder rural en la sociedad española de la Restauración (1874-1902)", Anales de la Universidad de Alicante. Historia contemporánea 7 (1989. 90), pp. 25-36. En una visión tópica de los conflictos y realidades del poder local se asientan buena parte de los trabajos de los historiadores adscritos a esta línea metodológica, como $\mathrm{T}$. CARNERO: (La modernización del Pais Valenciano durante la Restauración» en JL GARCi $\Lambda$ DEJGADO (dir): España entre dos siglos (1875-1931). Continuidad y cambio, Madrid, Siglo XXI, 1991, pp. 251-275. Vid. también T. CARNERO, J. PALAFOX: Creixement, politització i canvi social 17901980, Valencia, 1990.

2 Una exposición de los planteamientos de este sector, en J.A. PIQUeras: (El abuso del método, un asalto a la teorías en S. CASTIL (coord.): La historia social en España. Actualidady perspectivas, Madrid, 1991, pp. 87-110; vid. las atinadas observaciones de S. JULIA: «La historia social y la historiografia española», Ayer 10 (1993), pp. 29-46, esp. p. 42.

3 P. Ruz: rConsideraciones críticas sobre la nueva historiografia valenciana de los años 60 y 70 s en J. AZAGRA, E. MATEU, J. VIDNL (eds.): De la sociedad tradicional a la economía moderna. Estudios de bistoria valenciana contemporánea, Alicante, 1996, pp. 15-33.

Hapsaní, I.IX/1, núm. 201 (1999) 51-58 
País Valenciano, lejos de las considetaciones teleológicas de ese fenómeno que son habituales en gran parte de la historiografia española ${ }^{4}$.

\section{La ruptura revolucionaria}

Así, puede que el resultado final de la revolución fuera un régimen liberal extraordinariamente oligárquico, como subraya la tendencia interpretativa dominante, pero ello no debe ocultar el profundo carácter de ruptura política que conllevó el proceso revolucionatio. Ni el debate sobre el principio de soberanía nacional es ajeno a la fragmentación política posterior de los nuevos grupos dominantes ni el liberalismo debe ser concebido como una ideología de clase, sino como un lenguaje político universalista susceptible de apropiación interpretativa por los más variados grupos sociales 5 .

Tampoco puede afirmarse que la revolución fuera irrelevante desde el punto de vista de la transformación social ${ }^{6}$. En el País Valenciano, los grupos dominantes vieron profundamente alterada su composición y cohesión interna. La nueva burguesía agraria encabezó un complejo proceso de cambio agratio con adaptación a la demanda internacional, que se ptodujo en un marco social caracterizado por una acentuada desposesión campesina, la primacía del arrendamiento y la importancia del préstamo usurario. La respuesta campesina consistió, al menos en las zonas de regadío, en una peculiar combinación de producción para la subsistencia y aprovechamiento de las nuevas posibilidades de comercialización agtaria. Las peculiaridades de la agricultura valenciana marcaron un giro conservador de los intereses acomodados, pero también la expectativa, a largo plazo, de la consolidación de la unidad familiar campesina a través de la fragmentación progresiva de la propiedad desde los años finales del siglo XIX. Ese afianzamiento de determinados sectores campesinos podía

4 Puede encontrarse una valoración de las aportaciones valencianas al debate en I. CASTELS: «La rivoluzione liberale spagnola nel recente dibattito storiografico», Siudi Storici 36-1 (1995), pp. 127-161. Vid. también M. MARTi: «La revolució liberal en perspectivan, Recerques 28 (1994), pp. 97-103.

5 M.C. ROMEO: Entre el orden y la revolución. La formación de la burguesía liberal en la crisis de la monarguia absoluta (1814-1833), Alicante, 1993 y «La sombra del pasado y la expectativa de futuro: «jacobinos», radicales y republicanos en la revolución liberab» en I. CASTELLS, L. RoURA (eds.): Revolucion y democracia. El jacobinismo europeo, Madrid, 1995, pp. 107-138. 1. BuRDIEL: La politita de los notables. Moderados y avanzados durante el régimen del Estatuto Real (183436), Valencia, 1987 y «Dret, compromís $\mathrm{i}$ violència en la revolució burgesa», Recergutes 22 (1989), pp. 77-101.

6 En este párrafo se sigue la interpretación de J. MiLLAN: «Els inicis revolucionaris de la societat valenciana contemporània. Revolució, canvi social i transformacions econòmiques, 1780-1875» en J. AZAGRA, E. MATEU, J. VIDAL, op. cit., pp. 125-162, articulo al que remitimos, además, por sus referencias bibliográficas. 
ir acompañado de su inclusión en las redes del catolicismo social ${ }^{7}$, lo que no siempre descartaba roces con las estructuras de poder locales. El consenso deferencial de los grupos agrarios menos favorecidos era aún menos consistente, como no lo era, desde luego, el que reflejan las actitudes de los sectores vinculados a la manufactura y al peculiar crecimiento urbano.

\section{Discontinuidad y permanencia durante la segunda mitad del siglo XIX ${ }^{8}$}

Es en este marco, y por lo que se refiere a la segunda mitad del siglo XIX, en el que cabe plantear las siguientes cuestiones:

1) El problema de las estructuras de poder local ha de enfocarse necesariamente desde la perspectiva de la continuidad, o hay que poner énfasis en los procesos de ruptura político-social ocurridos durante los siglos XIX y XX?

2) ¿Las discrepancias entre grupos acomodados son enfrentamientos episódicos e irrelevantes, o son susceptibles de una interpretación social de mayor entidad?

3) ¿Los grupos sociales subalternos son siempre sujetos pasivos de la dominación, o sus actuaciones, autónomas o no, juegan algún papel en la evolución de esas estructuras locales de poder?

En relación con estas preguntas, la hipótesis que manejamos es la siguiente:

1) Contra la tendencia a sobrevalorar los aspectos de continuidad en las estructuras de poder local, lo que vale la pena subrayar es la frecuente discontinuidad, si el problema se examina comparativamente en ámbitos territoriales diversos y en periodos cronológicos que superen el corto plazo. El análisis del poder local, que suele interpretarse como producto de la inmovilidad de la sociedad burguesa, puede plantearse exactamente a la inversa: como una manifestación, en la esfera política, de la movilidad social de un mundo liberal que tuvo su origen en una traumática ruptura revolucionaria.

2) Contra el hábito de simplificar la complejidad de las situaciones sociales efectivas, es preciso distinguir el diferente significado social de los grupos acomodados, que suelen set rotulados bajo conceptos más o menos generalizadores, como "burguesía», "clase dominante», "oligarquía», etc. Es cierto que las etiquetas partidistas adoptadas por sectores de esos grupos (especialmente durante la Restauración, en la que menudeaban los pactos tácitos o explícitos entre los dirigentes dinásticos) eran, a veces, incongruentes. Pero eso no quiere

7 S. GARrido: Las trabajadores de las derechas, Castellón, 1986; E/ sindicalisme catolic a la Safor, 1900-1936. Catolicisme social i politic en una comarta del Pais Valencià, Gandia, 1987; Treballar en comi. El cooperativisme agrari a Espanya (1900-1936), Valencia, 1996.

8 El epígrafe que sigue se basa en la comunicación presentada por el autor al II Congrés Internacional d'Història Local de Catalunya (Batcelona, 1993) y parte del análisis de diversos ejemplos locales en el átea lingǘstica catalana; se encuentra publicada como «Elogi de la discontinuitat. El poder polític local entre els segles XIX i XXus en Actes de/ II Congrés Internacional d'Historia Local de Catalunya. Formes $i$ relacions del poder local a l'epoca contemporània, Barcelona, L'Avenç, 1995, pp. 271-283.

Hipantia, LIX/1, núm 201 (1999) 51-58 
decir que, bajo esas banderas convencionales, no hubiera una lucha real entre segmentos de los grupos acomodados. Una lucha que, muy a menudo, correspondía al enfrentamiento, la substitución y el desplazamiento de grupos burgueses, variados en su origen social, la época de su consolidación, sus opciones económicas y socio-políticas, sus valores culturales, etc. No es suficiente comprobar que todos los dirigentes dinásticos eran «propietarios» o «ricos»; hay que dilucidar, también, si representaban "promociones» burguesas distintas.

3) Finalmente, es necesario concébir el poder local no sólo como un asunto privativo de las clases dominantes. En primer lugar, se ha de demostrar que la aceptación pasiva e indiscriminada de la dominación era la tónica general entre una población subalterna desmovilizada, o si existían posibilidades para la plasmación de fórmulas culturales de resistencia, en el nivel de las ideas y/o en el nivel de las actuaciones prácticas. Cuando se habla de uhegemonías -o, más modernamente, de "control sociab»-, hay que definir a qué nos estamos refiriendo y si esos fenómenos ocurrían en la realidad social.

En segundo lugar, es preciso reconsiderar el papel jugado por los sectores sociales subalternos en la lucha por el poder local. Ese papel fue, a veces, determinante (o, por lo menos, con una incidencia decisiva) en la disputa entre fracciones dominantes. Y no siempre respondía a una actitud totalmente subordinada; por ejemplo, el liberalismo dinástico pudo haber sido, durante mucho tiempo, la única opción de izquierda al alcance de algunos sectores populares. Por fin, hay que reconsiderar si hubo actuación autónoma de los grupos subalternos en movimientos contrarios al liberalismo oligárquico, de carácter clasista o, más frecuentemente, interclasista de signo tepublicano.

En la mayor parte de los casos, la revolución de 1868 y el periodo que abrió aparecen como una experiencia determinante en la definición de las actitudes políticas de los distintos grupos sociales. Este no es momento para someter a crítica las diversas interpretaciones, generalmente en clave economicista y menospreciadoras del alcance sociopolítico de la ruptura que supuso la Gloriosa. Lo que parece evidente es que, más allá del fracaso a corto plazo del experimento democrático, la revolución del 68 representó la apertura de un espacio público, llamémosle (anacrónicamente) de centro-izquierda, que se convirtió en punto de referencia ineludible para la trayectoria política posterior.

1868 inició un proceso de desplazamiento: los sectores sociales y políticos que más se habían beneficiado de la revolución liberal tuvieron que compartir el poder político con nuevos grupos sociales (en algunos casos, incluso, lo perdieron). Ese nuevo espacio abierto en la frontera entre el liberalismo oligárquico y el democrático fue ocupado por nuevos aparatos políticos que respondían a un realineamiento de las alianzas y las tensiones sociales. En muchas ocasiones, esos cambios políticos reflejaban un proceso de sustitución dentro de los grupos acomodados, a la que no fue ajena, sino todo lo contrario, la actuación, autónoma o no, de grupos subalternos. La estabilización política global, asegurada por el régimen de la Restauración, favoreció que estos experimentos de cambio, basados generalmente en estrategias interclasistas, hubieran de adoptar, a menudo, un carácter acentuadamente localista, con ritmos y rasgos desi-

Mi.paniz, LIX/1, núm. 201 (1999) 51-58 
guales. Pero el cambio social y político abierto en 1868 continuó incubándose tras la fachada aparentemente inmóvil del sistema.

\section{El ejemplo castellonense: la mutabilidad continuada de las jerarquías Iocales}

La reiterada sustitución en el vértice sociopolítico y la complejidad de los mecanismos mediante los cuales se produjo pueden set ejemplificadas en la mayoría de las localidades valencianas sobre las que contamos con estudios detallados. Sin embargo, el caso de Castelló de la Plana ofrece una perspectiva especialmente adecuada por la dimensión cronológica de lo que (aún insuficientemente) conocemos 9

La destrucción, en 1707, de las instituciones forales (y, en especial, del régimen municipal propio) favoreció el ascenso de nuevos grupos sociales fieles al Borbón. Se trataba de familias que accedieron generalmente a los escalones más bajos del privilegio, que monopolizaron el nuevo poder político local y se convirtieton en (o se consolidaton como) propietarios que acudían a la explotación directa o, en todo caso, a vias plenamente contractuales.

El dominio de esta nueva oligarquía urbana no estuvo, sin embargo, exento de contestación desde abajo. En 1753 se llegó, incluso con referencias contratias al «despotismo», a la recogida de hasta 1.103 firmas en un intento exitoso de conseguir el rescate de las regidurías enajenadas. Los problemas relacionados con el control municipal del agua de riego (un fenómeno crucial en el regadío valenciano) se encontraban en la raíz de la protesta. Otras resistencias antioligárquicas tenían como vehículo los gremios, en especial la Cofradía de San Miguel (Gremio de Labradores desde 1818), que encuadraba a sectores medios del campesinado (sin excluir, sin embargo a los jornaleros). El trasfondo de esta conflictividad venía constituido por una creciente privatización de la tierra, en un proceso de acentuada diferenciación que, sin embargo, no desembocó en una polarización extrema. Por otra parte, el cultivo y elaboración del cáñamo, en expansión durante todo el XVIII, permitió la consolidación de una manufactura urbana pujante, hegemonizada por un grupo de maestros artesanos y de comerciantes, interesados en forjar un patrimonio en el área regada, cuya expansión se veía frenada por la amortización y la vinculación.

En el período revolucionario liberal, la élite de propietarios privilegiados fue incapaz de subordinar a los distintos estratos campesinos y a las fuerzas ur-

9 Este apartado se basa en la tesis de licenciatura de M. MArri: Un liberalismo de clases medias: el Ayuntamiento de Castello de la Plana en la época de la revolución liberal (1808-1858), Castellón, 1998, y en diversos trabajos del autor, en especial aLas raíces sociales del comportamiento político en la Restauración. Análisis de una élite política provinciana (Castelló de la Plana, 1875-1891) ", Estudios de Historia Social 54-55 (1991), pp. 443-467; «Resistència, crisi i teconstrucció dels republicanismes valencians durant els primers anys de la Restauració (1875-1891)", Recerques 25 (1922), pp. 73-101; $y$ «Los grupos agrarios en la política urbana del Pás Valenciano: Castelló de la de la Plana durante los siglos XIX y XX), Noticiario de Historia Agraria 11 (1996), pp. 57-79.

Hitşantia, 1.IX/1, núm. 201 (1999) 51-58 
banas emergentes. Por el contrario, la localidad, en un marco de crisis bélica aguda y reiterada, fue escenario de cierta movilización popular articulada por el nuevo lenguaje del liberalismo y manifestada en proyectos alternativos durante los momentos de ruptura política. La nueva élite liberal giró hacia la derecha tras 1845 , pero el «liberalismo respetable» castellonense no resultó hegemonizado por el moderantismo, sino por una oligarquía políticamente progresista, discontinua respecto de la anterior, que hizo compatible su afianzamiento económico, a través del arrendamiento y el préstamo, con un discurso mesocrático de referencias agraristas.

A partir de 1860, la mesocracia progresista, que también recurrió a prácticas clientelares, se encaminó hacia un enfrentamiento cada vez más abierto con el moderantismo. Lo hizo, sin embargo, a través de su encuadramiento en la Unión Liberal. Esta orientación facilitó la radicalización de un amplio sector de artesanos, tenderos y labradores modestos, apoyados en la movilización de los estratos más humildes, que hicieron del republicanismo federal la fuerza mayoritaria durante el periodo abierto por la revolución de 1868, contando, incluso, con el apoyo de ciertos elementos de la burguesía local.

Las tensiones políticas y sociales del Sexenio (la experiencia cantonal, especialmente) se saldaron con la dertota del republicanismo, que durante los primeros años de la Restauración hubo de concentrar sus esfuerzos en la recomposición de la alianza interclasista destruida durante el periodo democrático. Cuando lo logró, su embate electoral hizo entrar en crisis el dominio de los partidos dinásticos, lo que forzó a los grupos conservadores a recurrir a la movilización de determinados sectores del campesinado medio y precario, receptivos al mensaje y actuación del catolicismo social y político.

El trasfondo socioeconómico de la segunda mitad del siglo XIX contribuye a explicar esta compleja evolución política. Ante la larga crisis de la manufactura del cáñamo, los grupos acomodados teaccionaron mediante la configuración de una alternativa desarrollista centrada en la potenciación del factor capitalidad y en la consolidación de la agricultura de exportación. El fracaso relativo de la oligarquía liberal en el logro de estos objetivos llevó a algunos elementos pudientes a la confluencia con una movilización republicana que consideraban imprescindible para el desbloqueo de la situación política local. Por su parte, las capas populares se vieron ante la disyuntiva de incorporarse ventajosamente al proceso de transformación urbana promovido por los dirigentes republicanos o persistir, a cambio de cierta subordinación, en su propósito de consolidarse como pequeños propietarios agricolas.

Los sectores de profesionales y empresarios que acabaron encabezando las filas republicanas experimentaron, en las primeras décadas del siglo XX, una acentuada ascensión social que conllevó el desplazamiento gradual de la vieja oligarquía decimonónica. Sin embargo, la alianza interclasista republicana no se mostró inmune a los resultados del cambio que había promovido. Por una parte, surgieron cada vez con más fuerza círculos descontentos del control impuesto por los dirigentes republicanos tradicionales, que configuraton alternativas de izquierda (incluyendo el republicanismo valencianista). Por otra 
parte, la distancia creciente entre dirigentes y base social contribuyó al éxito gradual de las alternativas de clase, especialmente el sindicalismo socialista. Durante la Segunda República, el viejo republicanismo radical acabó por perder, a manos del Frente Popular, la reserva de voto obrero que le había permitido protagonizar la escena política local. El resultado de la guerra civil les arrebató cualquier posibilidad en este sentido.

\section{Consideraciones finales}

Los resultados obtenidos no deben ocultar las deficiencias con las que topa todavía nuestro conocimiento de las estructuras del poder local durante el siglo XIX. En primer lugar, se mantiene una relativa ignorancia sobre la política rural, dado que la mayor parte de los estudios disponibles se centran en las capitales y, en todo caso, en la importante red de ciudades de tamaño medio que constituye un rasgo esencial de la trayectoria histórica del País Valenciano.

En segundo lugar, llama la atención el vacío historiográfico sobre las décadas centrales de la centuria, incluyendo el episodio capital de 1868, sin el cual, como hemos intentado exponer, cualquier explicación que confronte la conflictividad de comienzos de siglo con la aparente estabilidad de la Restauración resulta todavía muy arriesgada.

Por último, y para la segunda mitad del XIX, se echa en falta la ligazón entre el estudio de los mecanismos de la política local y la dinámica general, aunque los trabajos sobre las diputaciones provinciales durante la Restauración ofrecen elementos interesantes de análisis ${ }^{10}$. La incidencia en este aspecto podría contribuir a un diálogo fructífero con otras tradiciones interpretativas.

Sin embargo, los déficits apuntados tampoco deben oscurecer lo que considero logros relevantes: la importancia del análisis cronológico a largo plazo y un insistencia destacable en la interpretación social de la política. Esa insistencia arranca, sin duda, de la influencia perdurable, entre sectores importantes de la historiografia valenciana, de la obra de E.P. Thompson. La difuminación de las fronteras entre campos antaño separados desde el punto de vista analítico se ve entiquecida, para algunos de nosotros, por las reflexiones sobre los fenómenos de hegemonía (concebidos como conjunción de coerción y consenso) de, por ejemplo, autores como J. Scott, J. Habermas, J. Kocka, A. Lüdtke o H. Medick.

10 A. YANINI: «Els cacics rurals valencians i la seva oposició a la guàrdia civil com a guarderia rural (1876-86)", Estudis d'Història Agrària 5 (1985), pp. 115-132; R. ZuRITA: Notables, politicos y clientes. La politica conservadora en Alicante (1875-1898), Alicante, 1996; M. MarTí «Las diputaciones provinciales en la trama caciquil: un ejemplo castellonense durante los primeros años de la Restauracións, Hispania 179 (1991), pp. 993-1041. Con una orientación muy distinta, M. Ciusr (dir.): Historia de la Diputación Provincial de Valencia, Valencia, 1995.

Vlippania, I.IX/1, núm. 201 (1999) 51-58 